\title{
A propos de : Sociétés, développement et santé, coordination Didier Fassin et Yannick Jaffré, Paris, Ellipses/Aupelf, 1990 pp.287.
}

Michael Singleton

\section{(2) OpenEdition \\ Journals}

Édition électronique

URL : http://journals.openedition.org/apad/394

DOI : 10.4000/apad.394

ISSN : $1950-6929$

Éditeur

LIT Verlag

Édition imprimée

Date de publication : 15 mars 1992

\section{Référence électronique}

Michael Singleton, «A propos de : Sociétés, développement et santé, coordination Didier Fassin et Yannick Jaffré, Paris, Ellipses/Aupelf, 1990 pp.287. », Bulletin de l'APAD [En ligne], 3 | 1992, mis en ligne le 06 juillet 2006, consulté le 23 septembre 2020. URL : http://journals.openedition.org/apad/394 ; DOI : https://doi.org/10.4000/apad.394

Ce document a été généré automatiquement le 23 septembre 2020.

Bulletin de I'APAD 


\title{
A propos de : Sociétés, développement et santé, coordination Didier Fassin et Yannick Jaffré, Paris, Ellipses/ Aupelf, 1990 pp.287.
}

\author{
Michael Singleton
}

1 Ceux qui, comme moi, étant chargés d'un cours d'anthropologie sociale de l'Afrique dont les consommateurs les plus avertis et assidus sont des membres de l'Ordre Médical établi par l'Occident au Sud, cherchent désespérément une entrée en matière à la fois sociologique et sanitaire. Qu'ils ne cherchent plus, car ils trouveront dans cet ouvrage la pierre du proverbe anglo-saxon qui tue deux oiseaux d'un seul coup. Il y a des bonnes monographies (une des dernières en date étant Santé et Pouvoir de D. Fassin), il y a des numéros spéciaux de revues (le $\mathrm{n}^{\circ} 28$ de Politique Africaine, par exemple), il y a des ouvrages monumentaux (on pense à L'anthropologie de la maladie de Laplantine), mais tout cela est soit trop codé pour des débutants, soit trop ciblé pour des simples sympathisants du dehors. Par contre les textes rassemblés par Fassin et Jaffré coalescent dans un tout qui combine théorie et pratique, métaphysique et méthode - et tout ça en fonction du développement.

Mais c'est justement là que le bât blesse un peu, car le développement en question est surtout celui de l'Afrique. Or la question même du développement ne devrait-elle pas être posée aussi bien au Nord qu'au Sud, que ce soit en général ou du côté des philosophies et des pratiques alternatives de la santé ? S'insérant dans une collection "Médecine tropicale", le livre abonde dans le sens d'une dichotomie qui oppose les "déjà développés" à ceux qui ne le sont pas encore. Il pourrait même donner l'impression que les anthropologues-africanistes s'intéressent à des ethnomédecines là où les sociologues-européens s'occupent de La Médecine tout court. Mais localiser des maladies dans les tropiques, n'est-ce pas euphémiser le fait qu'on y est malade parce que, en plus des moustiques, le désordre international est malsain pour les condamnés de ces terres-là ? D'autre part, cette Médecine qu'on a (in)consciemment déculturalisée 
pour mieux l'opposer à des ethnomédecines n'est-elle pas en fait l'approche médicale propre à l'ethnie occidentale ? Toute médecine n'est-elle pas une ethnomédecine ? Mais même cette prémisse prête à confusion dans la mesure où la médecine, étant un phénomène spécifique au social-historique de l'Occident, les autres gestions de la santé seraient légitimement à la fois plus et moins que proprement médical. On ne voit pas pourquoi (ou plutôt on voit très bien pourquoi!) la bio-médecine scientifique ferait figure d'étalon ou d'apogée en matière de bien être dans sa peau et en société. Si les différents systèmes de parenté ne sont pas des variations accidentelles d'une Famille archétypique, il n'y a pas de raison que des champs phénoménaux classés comme médicaux doivent faire référence à une substantialité sous-jacente... nouménale ou naturelle.

3 Mais même si vous n'êtes pas chargé d'un cours d'anthropologie de la santé, en tant que "simple" apadien(nne) vous avez tout intérêt à lire ce livre, ne serait-ce que pour poser la question de l'impact d'une anthropologie du changement social sur le développement euro-africain. Car le problème du rapport entre les résultats des anthropologues et les réalisations des acteurs médicaux ("patients" inclus) reste entiers. En général, comment se fait-il que les "choses" construites par nos recherches-actions anthropologiques deviennent si rarement des réalités concrètes? D'un côté, les propositions des experts que nous sommes, paraissaient à ce point plausibles et praticables qu'elles devraient s'imposer d'elles-mêmes, tandis que de l'autre, non seulement on n'a pas l'air de pouvoir, sinon de vouloir comprendre, mais, au mieux, on se montre indécis ou, au pire, on agit en dépit du bon sens scientifique. Ainsi, à moins que ce soit son homonyme, le professeur Marc Gentilini qui préface ce livre aux accents populaires et parallèles, est celui-là même qui, lors d'un colloque de Bamako (20 21.12.1989 - à ne pas confondre avec celui qui aboutissait en 1988 à l'Initiative de Bamako !) aurait déclaré : "l'esprit d'Alma Ata a, entre autres, contribué à faire perdre deux ans à l'OMS dans sa lutte contre le sida. Alma Ata n'était au fond que la résultante de tout ce qui allait dans le sens des démarches populaires pseudo-démocratiques, dans le sens du tout pour le peuple, rien que pour le peuple. Finalement, c'est un fiasco, comme celui des régimes qui développaient ces idées" (Le Monde, 28.12.1989). Hors de la biomédecine, hors de son personnel coûteux et de ses recherches scientifiques, point de salut! Lors du Forum des Partenaires, organisé par l'ORSTOM à Paris en septembre 91, je m'étais inscrit, seul anthropologue parmi des dizaines de praticiens, dans l'atelier sur la médecine... mais il était surtout question de marketing médical et très peu du peuple comme parti prenant.

Dans une autre incarnation plus catholique que la présente, j'ai eu pas mal affaire aux questions de la polygamie des fidèles et du célibat des prêtres en Afrique. Rien de dirimant, du point de vue théologique, n'interdisant l'une et l'autre de ces pratiques, une quasi-unanimité s'était fait jour parmi les missiologues expatriés et leurs homologues "indigènes" pour les justifier tant aux yeux des autorités ecclésiastiques qu'aux oreilles pies des braves laïcs. Mais pour trente-six raisons qui me paraissent aujourd'hui plus raisonnables et légitimes que ce ne fut le cas à l'époque où je militais pour ces causes perdues, ni le clergé ni le peuple jugeaient qu'il était dans leur intérêt de suivre à la lettre la logique des experts.

5 Maintenant que je donne dans l'anthropologie de la maladie, je sais que ma bataille avec les médecins et leurs clients n'est même pas gagnée à $9 / 10$ par la simple présentation de mes enquêtes et de mes élucubrations dans le domaine de l'éco-santé. 
J'aurais beau soigner l'emballage de mes missives et camoufler mes missiles, ma guerre est perdue d'avance. Il ne s'agit pas de la justesse des théories anthropologiques et de l'impossibilité pratique qu'éprouveraient des praticiens harcelés par la politique du pire pour en tenir compte. La réflexion sur et/ou la réfection du monde sont d'abord fonction du lieu où on se trouve et ce n'est qu'après avoir soustrait tout ce qui relève de la loi "logique/langage/lieu" qu'on pourrait éventuellement passer à des jugements de valeur. C'est peut-être la seule chose qui "manque" à ce genre d'ouvrage : une prise de conscience de l'(in)communicabilité et l'(in)opérationalité croissantes de son propre discours au fur et à mesure qu'on s'éloigne de son lieu de sa production... un manque que l'APAD pourrait combler? 